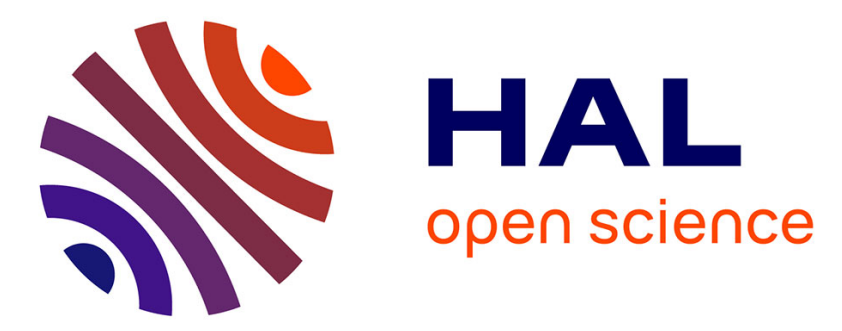

\title{
Pointage manuel et vocal : corrélats sensorimoteurs et neurocognitifs, coordination geste manuel / parole
}

Marion Dohen, Hélène Loevenbruck, Benjamin Roustan, Coriandre Emmanuel Vilain

\section{- To cite this version:}

Marion Dohen, Hélène Loevenbruck, Benjamin Roustan, Coriandre Emmanuel Vilain. Pointage manuel et vocal: corrélats sensorimoteurs et neurocognitifs, coordination geste manuel / parole. Louis-Jean Boë, Jean-Luc Schwartz. La parole. Origines, développement, structures, Editions Ophrys, pp.97-116, 2011, Faits de Langues, n³7, 978-2-7080-1314-8. hal-00784592

\section{HAL Id: hal-00784592 \\ https://hal.science/hal-00784592}

Submitted on 19 Sep 2013

HAL is a multi-disciplinary open access archive for the deposit and dissemination of scientific research documents, whether they are published or not. The documents may come from teaching and research institutions in France or abroad, or from public or private research centers.
L'archive ouverte pluridisciplinaire $\mathbf{H A L}$, est destinée au dépôt et à la diffusion de documents scientifiques de niveau recherche, publiés ou non, émanant des établissements d'enseignement et de recherche français ou étrangers, des laboratoires publics ou privés. 


\title{
Pointage manuel et vocal : corrélats sensorimoteurs et neurocognitifs, coordination geste manuel / parole
}

\author{
Marion Dohen, Hélène Lœvenbruck, \\ Benjamin Roustan et Coriandre Vilain*
}

La parole ne peut se réduire à sa composante vocale. Lorsque nous parlons, nous bougeons en effet non seulement nos articulateurs mais aussi notre corps dans son intégralité et ces gestes participent de l'acte de communication (e.g. Kendon, 2004 ; McNeill, 1992, 2000). Un détour par la phylogenèse et l'ontogenèse confirme d'ailleurs l'enracinement profond de la gestualité manuelle dans la communication parlée.

Dans une perspective évolutionniste, les théories défendant une origine vocale du langage s'opposent à celles défendant une origine gestuelle. Les premières soutiennent que la parole et le langage dériveraient des cris d'animaux tels que les cris d'alarmes. Il apparaît en effet que les vocalisations animales, et notamment celles des primates non-humains, revêtent des propriétés pouvant leur conférer le statut de véritables précurseurs du langage humain. Les travaux de Zuberbühler et collègues indiquent que les cris de certains grands singes peuvent être considérés comme des signifiants, et que leur combinaison semble elle aussi contrôlée et informative (cf. Slocombe \& Zuberbühler, 2006 ; Arnold \& Zuberbühler, 2006). Lemasson et Barbu (présent volume) apportent des preuves que les vocalisations des cercopithèques présentent des propriétés sémantiques et proto-syntaxiques, qu'elles ne sont pas figées et qu'elles évoluent (aux niveaux production et perception/compréhension) notamment en fonction de l'habitat et du contexte social de communication. Il existerait ainsi une certaine forme d'apprentissage et d'adaptabilité sociale. Dans le cadre de la théorie "Frame then Content " (cadre puis contenu) et par extension de l'ontogénèse à la phylogénèse, MacNeilage (1998; MacNeilage \& Davis, 2000) argumente que le contrôle moteur des articulateurs utilisés dans la mastication aurait mené à la parole et au langage. Ces travaux vont ainsi dans le sens d'une base gestuelle orofaciale pour le langage. D'un autre côté, les théories sur l'origine gestuelle du langage proposent que la main a été utilisée pour la communication avant la bouche. Corballis (2003) argumente ainsi que les primates non-humains produisent des vocalisations involontaires alors qu'ils contrôlent et comprennent certains gestes manuels qui sont d'ailleurs souvent produits en dyades, à l'inverse des cris. Il y aurait d'abord eu une communication * Département Parole et Cognition, GIPSA-lab, CNRS UMR 5216, Université de
Grenoble 
sous une forme gestuelle qui aurait mené à un contrôle plus complexe des articulateurs et finalement à une forme de communication vocale (qui libérait ainsi les mains pour l'utilisation des outils par exemple). Arbib $(2003,2005)$ et Holden (2004) proposent quant à eux, une co-évolution des systèmes manuel et vocal vers le langage et la communication. Cette proposition s'inscrit d'ailleurs dans la lignée des observations de Iverson et Thelen (2003) pour l'ontogenèse qui ont montré que, chez les bébés, le contrôle moteur du conduit vocal est plus long à acquérir que celui de la main mais que les deux systèmes se développent en coopération. Vauclair (présent volume) souligne, au travers des études sur les primates non-humains, l'existence de substrats neuraux communs aux modalités manuelle et langagière pour la communication. Les similarités anatomiques notamment au niveau de l'assymétrie de certaines aires homologues à celles du langage chez l'homme ainsi que la latéralisation manuelle préférentielle limitée à l'exécution de gestes à vocation communicative chez les primates non-humains fournissent des arguments forts pour l'importance de la communication manuelle dans l'évolution de la parole chez l'être humain. Des études en neuroimagerie (Hubbard et al., 2009, Özyürek et al., 2007, Willems et al., 2007) montrent d'ailleurs que les aires cérébrales impliquées dans l'interprétation et la compréhension des gestes manuels produits en même temps que la parole sont incluses dans celles intervenant dans la compréhension du langage articulé. D'autres montrent même que le système moteur de la main est activé en perception auditive seule mais uniquement pour des tâches linguistiques (Floël et al., 2003). Au-delà du débat sur l'origine de la parole, il semble que les gestes communicatifs manuels aient joué un rôle dans son évolution et qu'il existe un lien fondamental entre ces gestes et la parole. Dans cette perspective, on comprend l'importance que la coordination main/bouche a pu avoir dans l'évolution de la communication parlée.

$\mathrm{Si}$ on s'intéresse à l'ontogenèse, les gestes manuels jouent également un rôle important dans le développement du langage chez le bébé. Les gestes manuels permettent en effet aux enfants d'apprendre à parler. La production de gestes manuels est ainsi liée à des gains importants dans le développement du langage de 9 à 13 mois (Bates et al., 1979 ; Caselli, 1990). Le nombre de gestes manuels et de combinaisons geste-parole produit à 16 mois permet d'ailleurs de prédire de façon précise l'étendue de la production vocale à 20 mois (Morford \& GoldinMeadow, 1992 ; Volterra et al., 2005). Un certain nombre d'étapes clés dans le développement de la parole sont de plus liées à la production gestuelle : la combinaison d'un mot avec un geste véhiculant une information complémentaire (non redondante) ouvre par exemple la voie à la production d'énoncés de deux mots vers 18 mois (Capirci et al., 1996 ; Goldin-Meadow \& Butcher, 2003). De plus, cette association entre la parole et les gestes manuels dans le développement du langage n'est pas simplement perceptive i.e. les enfants ne produisent pas des gestes parce qu'ils les ont vu produits par les adultes. Les enfants aveugles produisent en effet aussi des gestes manuels pendant les phases d'apprentissage de la parole (Iverson \& Goldin-Meadow, 1998).

Il apparaît donc que les gestes manuels sont importants à la fois dans l'émergence de la faculté cognitive de parole chez l'être humain et dans le 
développement de la parole chez l'enfant. Ces regards croisés confirment donc l'importance de la gestualité manuelle dans la communication parlée. Mais si gestes manuels et parole sont combinés, ce n'est pas au hasard et il est important de comprendre comment. Dans cette perspective d'approfondissement de la multimodalité communicative gestes / parole, un cadre nous semble particulièrement intéressant : il s'agit de celui de la désignation. La désignation permet d'orienter l'attention de l'interlocuteur vers un objet, un endroit, une personne ou une entité abstraite afin que cet élément devienne l'objet de l'attention partagée des interlocuteurs. Il s'agit de pointer vers cet élément et ce pointage peut être réalisé à la fois avec les mains et avec la parole.

Le geste de pointage manuel est le plus souvent réalisé avec le bras étendu et l'index étiré dans la direction de l'élément intéressant, les autres doigts étant repliés à l'intérieur de la main (Butterworth, 2003). Il s'agit d'un geste manuel très important notamment parce qu'il joue un rôle particulier dans de nombreuses étapes du développement du langage (voir e.g., Goldin-Meadow \& Butcher, 2003 ; Pizzuto et al., 2005 ; Volterra et al., 2005). Les premiers gestes produits par les enfants vers 9-11 mois sont en effet des gestes déictiques (des gestes qui montrent) et leur production est prédictive de l'apparition des premiers mots. Le pointage joue de plus un rôle crucial dans le développement du lexique et de la syntaxe chez les bébés. Ce geste n'est cependant pas seulement utilisé par les enfants, il l'est aussi par les adultes. Notons que le pointage peut également être réalisé avec les yeux : c'est le pointage oculaire. Le regard s'oriente alors vers l'objet à désigner. Ce type de désignation est très précocement compris par les enfants (Lee et al., 1998).

Le pointage peut également être véhiculé par la voix : un élément de l'énoncé est mis en valeur afin de le désigner, de l'identifier et de le sélectionner parmi les autres éléments du discours. En français, la fonction de pointage est réalisée vocalement principalement grâce à la focalisation qui permet au locuteur de mettre en valeur la partie de l'énoncé qu'il veut faire passer comme la plus informative (cf. e.g., Gussenhoven, 1983 ; Halliday, 1967 ; Ladd, 1996). La focalisation permet ainsi de concentrer ou d'attirer l'attention de l'interlocuteur sur un élément particulier de l'énoncé qu'il s'agisse d'un mot ou d'un groupe de mots (Nølke, 1994). En d'autres termes, il s'agit de pointer vers l'élément de l'énoncé en question. La focalisation peut être réalisée principalement de quatre façons : grâce à la prosodie, à l'extraction syntaxique, à une combinaison des deux ou à l'usage d'un démonstratif (cf. Morel \& Danon-Boileau, 1992). La focalisation prosodique utilise un contour intonatif spécifique sur l'énoncé dans son intégralité et sur l'élément "pointé » en particulier : «MADELEINE m'amena ». Ce contour intonatif permet de mettre en valeur l'élément «pointé » (Madeleine dans l'exemple). L'extraction syntaxique implique l'utilisation d'une forme clivée qui isole l'élément pointé en début de phrase pour mieux le mettre en avant : «C'est Madeleine qui m'amena ». L'extraction syntaxique peut ou non être accompagnée de focalisation prosodique : «C'est MADELEINE F $_{\mathrm{F}}$ qui m'amena » (cf. Khaldoyanidi \& Morel, 2008 pour de telles observations en parole spontanée). Enfin, on peut également pointer en parole en utilisant un démonstratif: « Ce chien est noir ». 
L'importance du pointage dans le développement du langage aussi bien que sa nature multimodale donnent ainsi à penser que son étude nous apportera des informations cruciales sur les mécanismes mis en jeu dans la combinaison et la coordination entre gestes manuels et parole. Les buts de cet article sont donc : 1 . d'explorer les corrélats acoustiques, articulatoires et perceptifs du pointage vocal prosodique; 2. d'étudier les corrélats neuraux des différentes modalités du pointage afin de mieux comprendre comment elles sont liées entre elles; et enfin 3. d'explorer la coordination entre pointage manuel et vocal pour mieux comprendre comment main et parole montrent ensemble.

\section{CORRElats ACOUSTIQUeS, ARTICUlATOIRES ET PERCEPTIFS DU POINTAGE} PROSODIQUE

Nous nous intéressons dans cette partie à une des formes du pointage vocal qu'est la focalisation prosodique. L'intérêt est ici de comprendre comment les locuteurs «montrent» sans avoir recours à une structuration syntaxique facilement identifiable par l'interlocuteur. L'énoncé dont l'un des éléments est focalisé prosodiquement est en effet grammaticalement et syntaxiquement complètement identique à l'énoncé neutre. C'est donc grâce à l'utilisation exclusive de corrélats acoustiques et articulatoires que la désignation peut être ici véhiculée. Ce sont ces corrélats et leur perception que nous avons voulu explorer.

\subsection{Corrélats acoustiques}

Les corrélats acoustiques de la focalisation prosodique contrastive en français ont été décrits de façon exhaustive (voir e.g. Dahan \& Bernard, 1996; DelaisRoussarie et al., 2002; Di Cristo, 1998; Di Cristo \& Jankowski, 1999; Dohen, 2005 ; Dohen \& Lœvenbruck, 2004; Jun \& Fougeron, 2000; Rossi, 1999; Touati, 1987). La focalisation prosodique implique une augmentation de la fréquence fondamentale $(\mathrm{F} 0)$ et de la durée du constituant focalisé. Elle est aussi associée à une diminution de la F0 sur le constituant pré-focal et une désaccentuation des constituants post-focaux (contour de F0 plat). Au niveau métrique, Astésano (2001) postule, dans la lignée de Di Cristo (2000) que l'accent initial emphatique présent dans la focalisation contrastive est une hyper-réalisation de l'accent métrique initial secondaire en français. La focalisation s'accompagne également d'une augmentation de la durée du syntagme focalisé (particulièrement de l'attaque de la syllabe focalisée). On observe parfois aussi une augmentation anticipatoire de la durée de la syllabe pré-focale. Enfin, s'il n'existe pas de diminution de la durée des constituants post-focaux (pas de « dephrasing »), la focalisation peut entraîner une réorganisation prosodique de l'énoncé (« rephrasing ») par rapport au cas neutre : découpage d'un syntagme accentuel en deux pour isoler l'élément focalisé ou isolement de l'élément focalisé dans un syntagme intonatif propre (cf. Dohen, 2005 ; Dohen \& Lœvenbruck, 2004). Le pointage prosodique requiert donc un contrôle laryngé très fin de la part du locuteur. 


\subsection{CORRELATS ARTICULATOIRES ET PERCEPTIFS}

Nous présentons ici les résultats de deux études sur le français, menées dans notre département. La première concerne les mouvements de la langue. La seconde est un ensemble d'expériences sur les mouvements labiaux et faciaux. Des tests perceptifs ont permis de vérifier que les indices articulatoires repérés par ces études sont bien perçus visuellement.

1.2.1. Mouvements de la langue : Les mouvements de la langue ont été suivis à l'aide d'un articulographe, lors de la production de phrases simples dans différentes conditions de focalisation contrastive par une locutrice francophone (cf. Lœvenbruck, 1999). Une analyse prosodique a permis de montrer que dans tous les énoncés focalisés, le pic de F0 était porté par la syllabe initiale du syntagme focalisé. Les caractéristiques articulatoires de cette syllabe initiale en condition focalisée et neutre ont été comparées. Les données montrent que le déplacement de la langue est plus important pour cette syllabe en condition focalisée qu'en condition non-focalisée si elle appartient à un mot en position non-initiale dans l'énoncé (les syllabes en position initiale dans l'énoncé sont souvent articulées avec plus d'amplitude, même dans des contextes nonfocalisés, voir e.g. Fougeron \& Keating, 1997; Tabain, 2003). Les résultats des mesures pour la durée montrent que, pour cette locutrice, la durée de la syllabe initiale est plus élevée en condition focalisée que non-focalisée, quand la syllabe n'est pas à l'initiale de l'énoncé (les syllabes en position initiale d'énoncé sont souvent allongées, même sans focalisation). La focalisation prosodique semble donc, dans ce contexte, être associée à une hyper-articulation des mouvements de la langue sur la syllabe initiale (portant le pic de F0) de l'élément focalisée.

1.2.2. Indices labiaux: Dans un deuxième ensemble d'expériences, nous avons examiné les mouvements potentiellement visibles des lèvres durant la production de focalisation contrastive prosodique, en utilisant différentes techniques de mesure. Dans la suite, le cas neutre fait référence à un énoncé produit sans focalisation.

Etude vidéo - Les résultats résumés ci-dessous sont détaillés dans Dohen (2005). Les données ont été recueillies pour deux locuteurs (A \& B). Elles montrent que la focalisation prosodique est caractérisée par une augmentation (hyper-articulation) de l'aire intéro-labiale et de son pic de vitesse pour le constituant focalisé (par rapport à la fois au même constituant dans le cas neutre et au reste de l'énoncé focalisé). Le degré d'hyper-articulation varie avec le locuteur: A hyper-articule beaucoup plus que B. La protrusion labiale, mesurée seulement pour le locuteur B, est elle aussi hyper-articulée, et de façon plus importante que l'aire intéro-labiale. De plus, les données montrent que le locuteur B hypo-articule les séquences post-focales (réduction de l'aire intérolabiale, du pic de vitesse de l'aire intéro-labiale et de la protrusion par rapport à la fois au même constituant dans le cas neutre et au reste de l'énoncé focalisé) mais pas le locuteur A. De plus, les syllabes du constituant focalisé sont significativement allongées pour les deux locuteurs, particulièrement à l'initiale. 
Pour le locuteur A, la syllabe précédant le constituant focalisé est elle-même hyper-articulée et allongée, ce qui reflèterait une stratégie d'anticipation de la focalisation. Pour résumer, il semble qu'il y ait une tendance globale à l'hyperarticulation du constituant focalisé (par rapport à la fois au cas neutre et au reste de l'énoncé focalisé), mais que d'autres indices soient produits de façon complémentaire, et optionnelle.

Etude Optotrak - Le but de cette deuxième étude, présentée en détail dans Dohen (2005) et dans Dohen et al. (2006, 2009), était double. Il s'agissait d'une part de vérifier si les stratégies de production articulatoire visible de la focalisation prosodique identifiées chez les locuteurs A et B sont partagées par d'autres locuteurs. D'autre part, il s'agissait d'étendre les mesures faciales à d'autres paramètres que les lèvres : notamment les mouvements des sourcils et de la tête. Cinq locuteurs ont été enregistrés, en utilisant un système de suivi optique de marqueurs collés sur la face (Optotrak). Les résultats confirment qu'il existe bien des corrélats articulatoires potentiellement visibles de la focalisation contrastive prosodique en français et que la focalisation affecte l'énoncé dans son ensemble et pas simplement le constituant focalisé. La façon et le degré avec lequel les gestes articulatoires sont affectés par la focalisation dépendent du locuteur, mais on peut faire émerger deux stratégies :

- Stratégie absolue - Le constituant focalisé est allongé et hyper-articulé (aire intéro-labiale, protrusion labiale et mouvements de la mandibule plus amples par rapport à la fois au même constituant dans le cas neutre et au reste de l'énoncé focalisé). Les pics de vitesse peuvent également augmenter. Les locuteurs utilisant cette stratégie concentrent ainsi leur effort sur l'hyperarticulation du constituant focalisé. Certains peuvent même légèrement anticiper la focalisation.

- Stratégie différentielle - Le constituant focalisé est allongé et hyper-articulé (par rapport à la fois au même constituant dans le cas neutre et au reste de l'énoncé focalisé), mais dans une moindre mesure. La focalisation peut parfois être anticipée. La séquence post-focale est hypo-articulée par rapport au cas neutre. Un contraste visible est donc créé au sein de l'énoncé focalisé ce qui met en valeur l'élément focalisé au niveau articulatoire : l'hyper-articulation focale est peu importante mais elle est renforcée par une hypo-articulation post-focale.

Dans le cadre des deux stratégies, le locuteur met donc en place un contraste articulatoire potentiellement visible au sein de l'énoncé entre ce qui est focalisé et le reste.

Les résultats indiquent de plus qu'il pourrait bien y avoir un lien entre la focalisation prosodique et les mouvements de la tête (hochements) et des sourcils. Ces données sont à rapprocher d'autres études mentionnant des observations de pointage du menton (assimilable à un hochement de tête) en même temps que la focalisation en parole spontanée (Khaldoyanidi \& Morel, 2008). Nous avons néanmoins observé d'importantes variabilités inter- et intraindividuelles dans l'amplitude de ces mouvements et leur synchronisation avec le signal acoustique de parole. 
1.2.3. Tests perceptifs: Nous avons étudié la contribution des informations articulatoires et faciales mises en évidences pour la perception visuelle et audiovisuelle de la focalisation prosodique. Des tests perceptifs visuels indiquent qu'il est possible de détecter la focalisation prosodique uniquement à partir des données articulatoires visuelles (Dohen, 2005 ; Dohen \& Lœvenbruck, 2005). Une analyse croisée des résultats en production et en perception a permis de montrer que les indices perçus correspondent au moins en partie aux indices mesurés. De plus, deux tests perceptifs audiovisuels ont été menés sur de la parole chuchotée (dégradation des indices acoustiques prosodiques : la parole chuchotée n'a pas de fréquence fondamentale et contient donc peu d'informations intonatives) utilisée pour diminuer les performances en perception auditive de la focalisation prosodique (qui sont autrement excellentes). Les résultats (Dohen \& Lœvenbruck, 2009) montrent que les scores de détection de la focalisation augmentent quand on passe de la modalité auditive seule à la modalité audiovisuelle. Les indices articulatoires et faciaux identifiés par les études des données vidéo et Optotrak sont ainsi non seulement produits par les locuteurs mais ils sont aussi utilisés en perception.

\subsection{Des représentations sensorimotrices $d u$ pointage prosodique: vers la multimodalité}

Du point de vue de la production, nos données acoustiques et articulatoires indiquent que les locuteurs organisent leur phonation et leur articulation de façon à transmettre la focalisation prosodique. Du point de vue de la perception, les tests perceptifs audiovisuels montrent que ces traits phonatoires et articulatoires sont récupérés par les auditeurs/spectateurs et utilisés pour détecter le pointage prosodique.

Nous pouvons donc émettre l'hypothèse que des représentations sensorimotrices intégrées (auditives, visuelles et proprioceptives) sont nécessaires pour produire le pointage prosodique de façon appropriée. Ces représentations sont probablement formées via l'activation d'aires cérébrales associatives, telles que les régions temporales et pariétales. Or, les pointages manuel et oculaire semblent recruter eux-mêmes le cortex pariétal postérieur (associatif). En ce qui concerne le pointage manuel, il a été suggéré que des représentations spatiales, formées dans les régions pariétales postérieures et prémotrices frontales, pourraient fournir l'interface entre perception et motricité nécessaire à l'organisation des gestes de pointage (Kertzman et al., 1997 ; Lacquaniti et al., 1997; Vallar et al., 1997). Des études plus récentes des pointages manuel et oculaire suggèrent que le pointage avec le doigt et le pointage avec l'œil recrutent un réseau commun, plus ou moins latéralisé à gauche, incluant les champs frontaux de l'œil (Frontal Eye Field, FEF) et le cortex pariétal postérieur (Astafiev et al., 2003 ; Connolly et al., 2000; Hagler et al., 2007 ; Simon et al., 2002). Ces travaux sur les réseaux des pointages manuel et oculaire suggèrent la nécessité d'impliquer des réseaux associatifs multimodaux pour pointer. Associés à nos propres observations sur les propriétés sensorimotrices du pointage prosodique, ces résultats nous ont incité à explorer 
et comparer les corrélats cérébraux du pointage dans ses différentes modalités : vocale (prosodique et syntaxique), manuelle et oculaire.

2. LES CIRCUITS CEREBRAUX DES DIFFERENTES MODALITES DU POINTAGE (MANUELLE, OCULAIRE, PROSODIQUE ET SYNTAXIQUE)

Note: La figure en annexe fournit une cartographie des aires de Brodmann référencées dans cette section.

\subsection{Corrélats cérébraux du pointage vocal}

Nous avons mené une première étude en imagerie par résonance magnétique fonctionnelle (IRMf) sur le pointage vocal en français véhiculé soit par la focalisation prosodique soit par l'extraction syntaxique chez les adultes (Lœvenbruck et al., 2005). Les sujets devaient prononcer trois types de phrase isosyllabiques :

1. condition contrôle : "Madeleine m'amena"

2. condition pointage prosodique : "MADELEINE m'amena"

3. condition pointage syntaxique : "C'est Mad'leine qui m'am'na"

a.

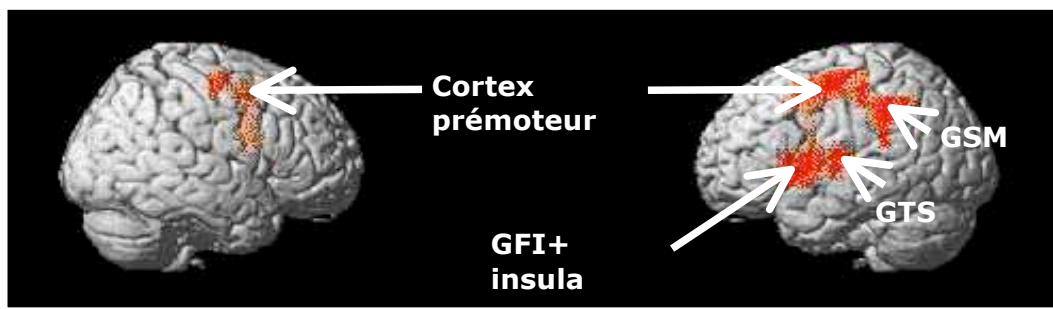

b.

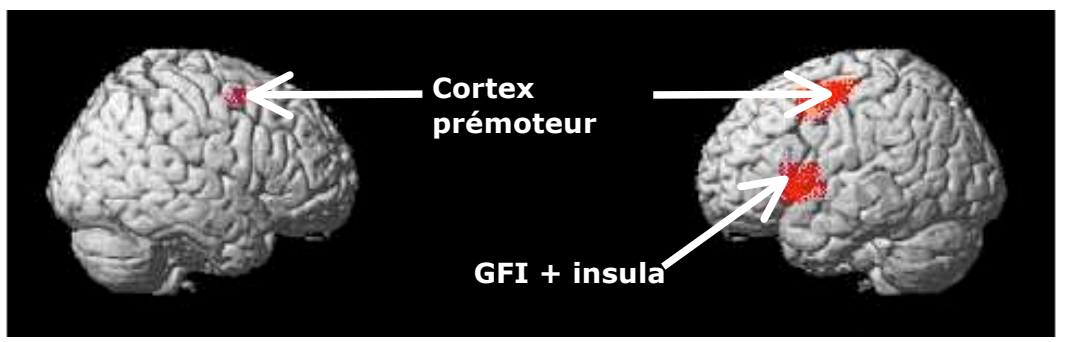

Figure 1 - Aires cérébrales activées dans a. la condition de focalisation prosodique par rapport à la condition de contrôle, b. la condition d'extraction syntaxique par rapport à la condition de contrôle. GFI : gyrus frontal inférieur (contient l'aire de Broca); GTS : gyrus temporal supérieur (contient l'aire de Wernicke) ; GSM : gyrus supramarginal, dans le lobule pariétal inférieur 
Nos résultats (cf. Fig. 1.a) montrent que le pointage prosodique recrute un réseau temporo-pariéto-frontal gauche comprenant :

- l'aire de Wernicke, impliquée dans la compréhension auditive ;

- le gyrus supramarginal (GSM) gauche, aire associative qui pourrait intégrer les sensations auditives et oro-laryngées ;

- le gyrus frontal inférieur (GFI) gauche, impliqué dans l'analyse de l'action (linguistique ou non).

Lors du pointage syntaxique, qui utilise une construction grammaticalisée, une dépariétalisation a été observée (cf. Fig. 1.b) : l'aire associative qu'est le GSM n'est pas activée. Il semble donc que le traitement intégratif sur les sensations auditives et somatosensorielles attendues disparaisse, tandis que demeure l'activation de Broca (BA 45, 47). La grammaticalisation serait donc pour nous une automatisation consistant à ne plus faire appel aux sensations attendues de sa propre action vocale pour focaliser.

\subsection{Corrélats cérébraux de différentes modalités de pointage}

Afin de mieux comprendre le lien fonctionnel entre les différentes modalités du pointage, nous avons mené une étude IRMf complémentaire visant à explorer les corrélats cérébraux de ces différentes modalités. Il apparaît que le circuit du pointage vocal est latéralisé à gauche (cf. ci-dessus). Or il semble que le pointage manuel recrute de façon prédominante le lobe pariétal gauche (Astafiev et al., 2003). Nous nous sommes ainsi posé la question des relations entre geste manuel de pointage et pointage vocal au niveau cérébral. Nos travaux ont porté sur la somatotopie du lobe pariétal lors du pointage avec divers effecteurs, l'œil, l'index et la voix. Quatre tâches ont été étudiées :

1. pointage syntaxique : prononcer « C'est Jules qui tient le livre».

2. pointage prosodique : prononcer « JULES tient le livre » avec focalisation prosodique sur Jules

3. pointage oculaire : diriger le regard vers Jules

4. pointage digital : tendre l'index vers Jules

L'objectif était de tester l'hypothèse que les différentes modalités du pointage pourraient activer des régions cérébrales voisines, notamment dans le lobe pariétal gauche. Les données enregistrées pour 15 sujets avec un scanner IRM ont été analysées statistiquement au niveau du groupe. Un certain nombre d'observations a ainsi pu être fait.

Le pointage manuel (par rapport à un geste manuel de contrôle) implique une activation bilatérale du lobule pariétal supérieur (BA 7), avec une prédominance gauche. On observe également une activation bilatérale du gyrus supramarginal (BA 40) avec prédominance droite. Une activation du cortex frontal gauche est également observée (BA 4, 6).

Dans la condition de pointage oculaire (par rapport à un contrôle oculaire), on obtient une activation prédominante gauche du gyrus postcentral (BA 3) et du 
lobe frontal bilatéralement (BA 4, 6). Les aires visuelles du gyrus occipital (BA $17,18)$ sont activées de façon bilatérale.

La condition de pointage prosodique (par rapport à la condition de parole neutre sans focalisation) recrute le lobule pariétal supérieur (BA 7) de façon bilatérale, avec une prédominance gauche. L'activation du gyrus supramarginal n'est pas significative contrairement à ce que nous avions obtenu dans Lœvenbruck et al. (2005). Le gyrus postcentral gauche est activé (BA 2,3) ainsi que les régions périsylviennes (BA 47, 13, 42), de façon bilatérale.

La condition de pointage syntaxique (par rapport à la condition de parole neutre) active le gyrus supramarginal droit (BA 40) mais ne recrute pas le lobule pariétal gauche. Les régions périsylviennes droites sont activées (BA 44, 47, 21), ainsi que le cortex prémoteur (BA 6) de façon bilatérale.

Pour résumer, cette analyse préliminaire de nos données révèle que le lobule pariétal supérieur gauche (BA 7 ou l'aire voisine BA 3) est activé pour trois modalités de pointage: manuel, oculaire et prosodique, mais pas pour le pointage syntaxique. Ainsi, ces trois modalités pourraient bien toutes recruter le lobule pariétal supérieur gauche, le pointage oculaire étant situé dans une zone plus antérieure que le pointage prosodique, lui-même plus antérieur que le pointage digital. Comme nous l'avons expliqué plus haut, les études comportementales (acoustiques et articulatoires) montrent que le pointage prosodique requiert un contrôle très précis du larynx et des articulateurs de la parole, ce qui suggère que les locuteurs utilisent des représentations multisensorielles (auditives et somato-sensorielles) pour produire le pointage prosodique de façon adéquate, tout comme ils le font pour produire un geste digital ou oculaire. Ces représentations seraient formées via l'activation d'aires associatives situées dans le lobule pariétal supérieur. L'absence d'activation pariétale lors du pointage syntaxique pourrait être due à l'absence de construction multisensorielle " en ligne » dans ce type de pointage vocal, par rapport au pointage vocal prosodique. Le pointage syntaxique utilise en effet une construction grammaticalisée, «prête-à-énoncer », et ne nécessiterait pas que des représentations intégrées (auditivo-articulatoires) soient formées.

L'ensemble de ces travaux confirme notre hypothèse que le pointage linguistique en ligne (la focalisation prosodique) est inscrit dans le même réseau cérébral que les pointages gestuels (manuel et oculaire). Pointages oculaire, manuel et vocal seraient ainsi bien liés, autant sur le plan développemental que sur le plan cérébral. La question de la coordination des pointages manuel et vocal fait l'objet de la suite de cet article.

\section{COORDINATION GESTE / PAROLE DANS LE POINTAGE}

\subsection{Etudes de la coordination geste / parole}

De nombreuses études ont analysé la coordination des gestes de la main et de la parole chez les adultes. Les deux types d'approches principalement utilisées sont des expériences "contrôlées » et des analyses de production spontanées ou semi-spontanées. Les expériences «contrôlées » visent à effectuer des mesures 
quantitatives précises pour évaluer les liens entre gestes manuels et parole. Elles impliquent l'utilisation de méthodologies contrôlées et des enregistrements dans des environnements «artificiels» tels que des plateformes expérimentales. L'analyse de productions spontanées permet d'étudier les liens entre gestes manuels et parole dans leur environnement naturel. Elles sont cependant difficiles à répliquer et ne permettent pas toujours d'effectuer des caractérisations quantitatives précises (elles reposent sur des descriptions détaillées d'observations uniques). Ces deux types de méthodes sont intéressantes et elles se complémentent parfaitement. Nous nous intéresserons ici cependant aux expériences « contrôlées ». Notre but était en effet d'obtenir une caractérisation phénoménologique plus précise des patrons généraux de coordination entre pointages manuel et vocal.

McNeill (1992) décrit la coordination gestes manuels / parole comme étant une synchronisation entre le geste manuel et le mot auquel il est rattaché sémantiquement et de façon co-expressive. Dans cette lignée de raisonnement et comme souligné par Rochet-Capellan (2007), il semble particulièrement intéressant d'étudier le pointage. Pour le pointage, la règle énoncée par McNeill, impliquerait une synchronisation entre le geste (qui montre) et la partie de la parole qui montre (déterminant, pointage vocal...). Levelt et collègues (1985) ont mené une étude dans laquelle ils demandaient aux participants de pointer vers une lampe en prononçant "cette lampe». Ils ont comparé les conditions parole seule, geste seul et parole et geste combinés. Ils ont observé un retard des réponses manuelles et vocales en condition geste et parole combinés (comparée aux conditions geste seul et parole seule). Ce retard était plus important pour la production vocale. Ces résultats ont ensuite été répliqués par Feyereisen (1997). Ces chercheurs ont conclu que la main et la bouche dépendraient de deux systèmes différents qui utiliseraient, en compétition, les mêmes ressources, l'un devant donc attendre que l'autre ait fini pour utiliser les ressources nécessaires à son fonctionnement. Ceci expliquerait, selon ces chercheurs, le délai mesuré pour les productions vocales en condition geste et parole combinés. Si l'on se place d'un point de vue légèrement différent, on pourrait proposer que ce délai pourrait être dû à des exigences de coordination: les productions vocale et gestuelle devant être synchronisées, la bouche attendrait la main pour que cette synchronisation soit possible. Un autre point important est que les études décrites ci-dessus mesuraient l'instant de déclenchement acoustique de la parole. Aucune autre mesure articulatoire ni acoustique n'était effectuée. Or si la coordination est de type moteur, se faisant donc plutôt entre la main et la bouche (en tant qu'articulateur), il semble inadéquat de mesurer un paramètre acoustique pour évaluer la réponse vocale à la tâche. Enfin dans les études décrites ci-dessus, la partie de la parole « qui montre » (le plus souvent un démonstratif) était toujours en début d'énoncé ce qui pourrait avoir un effet sur la coordination puisque, en suivant McNeill (1992), c'est avec cet élément que le geste serait synchronisé.

Rochet-Capellan et collègues (2008) ont mené une étude testant l'effet de la position de l'accent lexical en portugais au sein d'un non-mot de deux syllabes CVCV (e.g. /pápa/ vs. /papá/) sur la coordination entre la main et la mâchoire dans une tâche de pointage vers une cible. Un système de capture de mouvement 
(Optotrak) a été utilisé pour enregistrer les mouvements de la mâchoire et de l'index. Quand l'accent lexical se trouvait sur la première syllabe, l'apex du geste de pointer (position la plus étendue du bras et de l'index) était synchronisé avec l'ouverture maximale de la mâchoire correspondant à la syllabe accentuée. Quand l'accent lexical se trouvait sur la seconde syllabe, l'apex du geste de pointer se trouvait entre les deux pics d'ouverture de la mâchoire (voyelles /a/) et le geste de retour était synchronisé avec le maximum d'ouverture de la mâchoire correspondant à la syllabe accentuée. Ces résultats confirment que l'étude des gestes articulatoires pourrait être plus porteuse d'information que l'étude de l'instant d'initiation acoustique de la parole pour évaluer la coordination geste/parole. Ils montrent de plus que la position de l'accent lexical a un effet sur la réalisation du geste manuel.

\subsection{Coordination des gestes manuels et du pointage vocal prosodique}

Dans le prolongement des études présentées ci-dessus, nous avons mené une étude dont le but était d'explorer la coordination entre pointages manuel et prosodique pour des phrases complètes. Les questions posées étaient les suivantes : 1 . Comment les pointages manuel et vocal sont-ils coordonnés dans le temps? 2. Est-ce que cette coordination geste / parole est spécifique au pointage ? 3. La production du pointage manuel a-t-elle un effet sur la réalisation acoustique et articulatoire du pointage vocal ?

Nous avons utilisé un corpus composé de phrases de structure syntaxique sujet $(\mathrm{S})$ - verbe $(\mathrm{V})$ - objet $(\mathrm{O})$ en français avec une structure syllabique fixe (ex : Mumu tient le bébé). Nous nous sommes intéressés à deux types de focalisation prosodique : focalisations sur le sujet (FS) et sur l'objet (FO). Quatre conditions ont été étudiées : parole seule, pointage (geste déictique communicatif), geste de battement (geste non-déictique communicatif souvent lié à la prosodie dans la littérature) et geste contrôle d'appui sur un bouton (geste non-déictique et non communicatif). Une tâche de correction a été utilisée pour inciter à la production de focalisation prosodique dans un contexte d'interaction semi-naturelle. Deux images étaient affichées sur un écran en face du participant et il lui était demandé d'effectuer la tâche de correction tout en pointant vers la cible adéquate. Dans les conditions parole seule, geste de battement et geste de contrôle, les sujets effectuaient simplement le geste demandé en même temps qu'ils produisaient leur correction. Dix adultes droitiers de langue maternelle française ont participé à l'expérience ( 8 femmes et 2 hommes).

Les mouvements des lèvres et de la main droite ont été enregistrés grâce à un système $3 \mathrm{D}$ de suivi du mouvement (Optotrak). La parole était enregistrée grâce à un microphone.

Les frontières acoustiques des syllabes ont été annotées ainsi que les maxima de fréquence fondamentale $\left(\mathrm{F}_{0}\right)$ et d'intensité (Int) correspondant à l'élément focalisé ( $\mathrm{S}$ ou $\mathrm{O}$ ). Nous avons aussi analysé la durée (Dur) de l'élément focalisé. L'ouverture des lèvres et la protrusion de la lèvre supérieure ont été extraites des données de suivi du mouvement. Les cibles vocaliques $\left(\mathrm{CV}_{1}, \mathrm{CV}_{2}\right)$ correspondant à chacune des syllabes de l'élément focalisé (2 voyelles) ont ainsi 
été annotées (maxima d'ouverture des lèvres ou de protrusion). Concernant le mouvement du doigt, l'apex $\left(\mathrm{P}_{\mathrm{A}}\right)$ et le début du geste de retour $\left(\mathrm{P}_{\mathrm{R}}\right)$ ont été étiquetés. Pour le geste de pointage, l'apex correspond à la position la plus étendue de l'index vers la cible. Pour le geste de battement, l'apex a été identifié comme étant le point vertical le plus bas du mouvement. Pour le geste de contrôle, l'apex a été identifié comme étant le moment où l'index appuie sur le bouton. Les instants de réalisations de chacun de ces évènements (maxima de $F_{0}$ et d'intensité, $\mathrm{P}_{\mathrm{A}}$ et $\mathrm{P}_{\mathrm{R}}$ ) ont été normalisés sur la durée totale de l'énoncé afin d'éliminer les variabilités dues aux différences segmentales des énoncés et aux temps de réponse.

\subsubsection{Coordination temporelle geste / parole-Résultats généraux :}

Etude du geste manuel - Le type de focalisation a un effet significatif sur les instants de réalisations de $\mathrm{P}_{\mathrm{A}}$. Le geste a tendance à être réalisé plus tard au sein de l'énoncé quand l'objet est focalisé (FO) : on peut dire que la focalisation «attire » le geste. La condition gestuelle a également un effet significatif sur l'instant de réalisation de $\mathrm{P}_{\mathrm{A}}$. Ceci suggère que les différents types de geste ne sont pas réalisés de la même façon (en tout cas en ce qui concerne leur apex).

Etude de la parole - Le type de focalisation a un effet significatif sur tous les instants de réalisation des variables acoustiques et articulatoires. Ceci correspond au fait que les corrélats acoustiques et articulatoires ont été mesurés sur S pour FS et sur O pour FO. Ils arrivent donc forcément plus tard en condition FO. La condition gestuelle n'a d'effet significatif sur aucune des variables considérées. La production d'un geste n'a donc aucun effet sur l'organisation temporelle interne de l'énoncé focalisé.

3.2.2. Coordination temporelle geste / parole - Alignements temporels "Dans le but d'étudier l'alignement potentiel des gestes manuels avec la parole, nous avons calculé, pour chaque énoncé, les différences entre les instants de réalisation normalisés des évènements gestuels $\left(t_{P A}\right.$ et $\left.t_{P R}\right)$ et les instants de réalisation des évènements acoustiques $\left(t_{\mathrm{F} 0}\right.$ et $\left.t_{\mathrm{Int}}\right)$ et articulatoires $\left(\mathrm{t}_{\mathrm{CV} 1}\right.$ et $\left.\mathrm{t}_{\mathrm{CV} 2}\right)$. Nous avons ensuite calculé la moyenne de ces différences sur tous les énoncés pour chaque participant. La figure 2 donne les résultats du calcul des moyennes et écart-types sur tous les sujets (si une « boîte » est proche de zéro, cela signifie que les deux évènements sont proches dans le temps).

Le type de focalisation a un effet significatif sur toutes les différences temporelles. Quel que soit le type de geste, la coordination temporelle entre la parole et les gestes manuels au sein de l'élément focalisé est différente pour FS et FO. La condition gestuelle a également un effet significatif sur toutes les différences temporelles pour $\mathrm{P}_{\mathrm{A}}$. Les différents gestes manuels sont donc coordonnés à la focalisation prosodique de façons différentes : $\mathrm{P}_{\mathrm{R}}$ se trouve à peu près au même endroit relativement aux corrélats acoustiques et articulatoires mais pas $\mathrm{P}_{\mathrm{A}}$. 

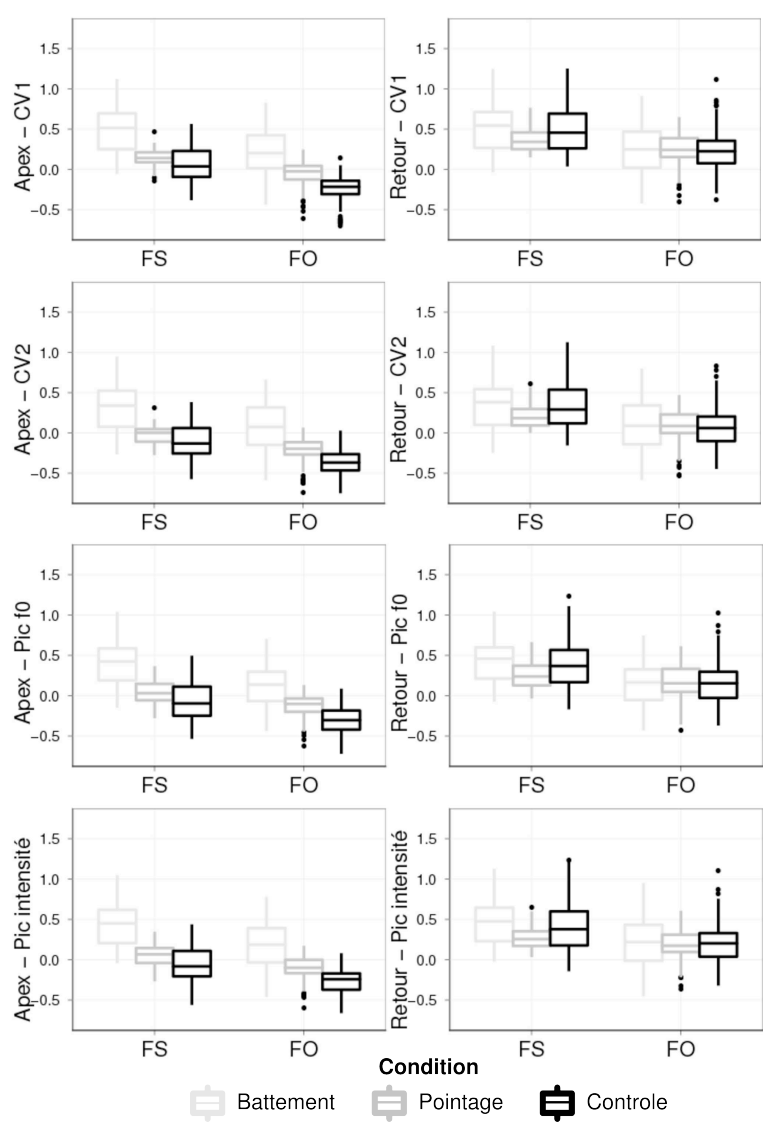

Figure 2 - Différences entre les instants normalisés de réalisation des évènements gestuels (Apex et Retour) et articulatoires $\left(\mathrm{CV}_{1}\right.$ et $\left.\mathrm{CV}_{2}\right)$ et acoustiques (pics de $\mathrm{F}_{0}$ et d'intensité) pour les différents types de focalisation et pour les différentes conditions gestuelles. Mode de représentation: Les barres à 0 représentent une différence nulle. Chaque rectangle représente les données correspondant à une condition (premier au troisième quartile), le trait représente la médiane. Les segments représentent l'étendue séparant le $5^{e}$ et le $95^{e}$ centiles et les points représentent les données en dehors de cette zone (" outliers»).

Nous avons ensuite comparé les temps normalisés correspondant à la réalisation des différentes variables gestuelles $\left(\mathrm{P}_{\mathrm{A}}\right.$ et $\left.\mathrm{P}_{\mathrm{R}}\right)$, acoustiques $\left(\mathrm{F}_{0}\right.$ et Int) et articulatoires $\left(\mathrm{CV}_{1}\right.$ et $\left.\mathrm{CV}_{2}\right)$ et ce pour chaque type de geste séparément (puisqu'il y a un effet de la condition gestuelle) et pour chaque type de focalisation séparément (puisqu'il y a un effet du type de focalisation).

Geste de pointage - On observe une tendance claire vers un alignement entre l'apex du geste de pointage avec une cible articulatoire de l'élément focalisé (FS: $t_{P A}$ 'est pas significativement différent de $t_{\mathrm{CV} 2} ; \mathrm{FO}: t_{\mathrm{PA}}$ n'est pas significativement différent de $t_{\mathrm{CV} 1}$ ).

Geste de battement - Pour FS, il n'y a aucune tendance vers un alignement entre $\mathrm{P}_{\mathrm{A}}$ et un des corrélats acoustiques ou articulatoires de la focalisation. Pour $\mathrm{FO}, \mathrm{P}_{\mathrm{A}}$ semble être aligné avec les maxima de $\mathrm{F}_{0}$ et d'intensité ainsi que $\mathrm{CV}_{2}$. 
Geste de contrôle - Pour FS, $\mathrm{P}_{\mathrm{A}}$ semble être aligné avec les maxima de $\mathrm{F}_{0}$ et d'intensité. Ceci n'est cependant pas le cas pour FO. Pour FS, $\mathrm{P}_{\mathrm{A}}$ est aligné avec $\mathrm{CV}_{1}$. Pour FO, $\mathrm{P}_{\mathrm{R}}$ est aligné avec $\mathrm{CV}_{2}$.

De façon très intéressante, la figure 2 montre aussi que les alignements sont plus précis et constants d'un sujet à l'autre pour le geste de pointage (voir les écart-types). Ceci est d'autant plus vrai si l'on regarde les données concernant l'apex.

3.2.3. Réalisations acoustiques et articulatoires de la focalisation - Effet de la production d'un geste manuel et de son type : Nous avons analysé les amplitudes des corrélats acoustiques (durée de l'élément focalisé et maxima de $F_{0}$ et d'intensité) et articulatoires $\left(\mathrm{CV}_{1}\right.$ et $\left.\mathrm{CV}_{2}\right)$. Le type de focalisation a un effet significatif sur toutes les variables. Il apparaît que les amplitudes de tous les corrélats acoustiques et articulatoires de la focalisation sont plus faibles en FO qu'en FS ce qui n'est pas surprenant. De façon plus intéressante, la condition gestuelle n'a d'effet significatif sur aucune des variables.

3.2.4. Bilan - Différents degrés de coordination geste / parole: le statut particulier du pointage : Les résultats de cette étude montrent que la focalisation et les gestes manuels sont coordonnés en ce sens que la focalisation « attire » le geste manuel. Ceci était prévisible pour le geste de pointage puisque les pointages manuel et vocal avaient le même objet qui était désigné au niveau vocal par la focalisation prosodique et au niveau gestuel par le pointage de l'index. Nous retrouvons donc bien là, dans la lignée des résultats de RochetCapellan et collègues (2008), que le geste manuel qui montre (pointage) et la partie de la parole qui montre (focalisation) se chevauchent. On trouve de plus que la coordination se fait essentiellement en alignant l'apex du geste avec une cible articulatoire plutôt qu'acoustique (comme Rochet-Capellan et al., 2008). L'attraction du geste manuel par la focalisation était également prévisible pour le geste de battement puisque plusieurs études ont déjà montré que le geste de battement était lié à l'emphase dans le discours. Par contre, nous ne nous attendions pas à un tel résultat pour le geste de contrôle pour lequel nous pensions qu'il n'y aurait aucune coordination particulière entre le geste manuel et la focalisation. Il est possible que le geste de contrôle ait été trop proche d'un geste de pointage (extension du bras et de l'index nécessaire pour aller appuyer sur le bouton). Cependant la coordination est beaucoup plus stricte pour le pointage que pour les autres gestes. Il apparaît donc que le lien fonctionnel entre geste et parole a une grande influence sur la coordination. C'est en effet pour le geste de pointage que ce lien est le plus fort. De plus, la coordination la moins stricte est observée pour le geste de contrôle qui est un geste non-communicatif.

Les résultats montrent aussi que la production de pointage manuel n'a aucun effet sur les corrélats acoustiques et articulatoires de la focalisation prosodique. Il n'y a aucune différence entre la condition parole seule et les conditions parole + geste et aucune différence non plus pour les différents types de gestes. Il semble donc que la production de pointage manuel n'affecte pas la production parallèle, et peut-être même conjointe, de pointage vocal prosodique. 


\section{CONCLUSION}

Le but de cet article était d'explorer le pointage dans son expression multimodale. Le pointage peut en effet être réalisé avec la main (et plus particulièrement l'index) ou avec la parole en utilisant la focalisation, exprimée grâce à la prosodie, à l'extraction syntaxique, à une combinaison des deux ou à l'usage d'un démonstratif.

La première partie visait à faire un bilan des corrélats acoustiques, articulatoires et perceptifs du pointage vocal prosodique (focalisation prosodique). Les observations effectuées nous ont conduits à émettre l'hypothèse que des représentations sensori-motrices très précises sont impliquées dans la production du pointage vocal prosodique. Le pointage prosodique requiert en effet une coordination très élaborée entre mouvements du larynx et des articulateurs de la parole, qui nécessite un contrôle en ligne auditif et somatosensoriel très précis. C'est dans cette perspective que nous avons jugé intéressant d'examiner les corrélats cérébraux des différentes modalités du pointage.

La deuxième partie visait ainsi à explorer et comparer les corrélats neurocognitifs de la production des pointages vocaux (extraction syntaxique et focalisation prosodique) et de plusieurs modalités du pointage (extraction syntaxique, focalisation prosodique, pointage oculaire et pointage manuel). Nos résultats renforcent notre hypothèse que le pointage vocal prosodique est ancré dans le même réseau cérébral que les pointages gestuels (manuel et oculaire). Par conséquent, geste de pointage manuel et pointage vocal seraient bien liés sur le plan cérébral ce qui corrobore l'hypothèse selon laquelle gestes manuels et parole se seraient co-développés dans l'évolution de la communication parlée.

Enfin, la troisième partie visait à explorer la coordination entre pointages manuel et vocal (prosodique) dans la production du pointage multimodal en comparaison à d'autres types de combinaisons geste / parole (focalisation prosodique + geste de battement ou geste contrôle non communicatif). Cette étude a permis une meilleure caractérisation de la coordination entre pointage vocal prosodique et gestes manuels (pointage manuel en particulier). Il est apparu que la coordination la plus fine était observée quand le geste manuel et la focalisation avaient un lien fonctionnel très fort (cas du pointage). Dans ce cas l'apex du geste était aligné avec des cibles articulatoires plutôt qu'acoustiques. On note donc encore une fois un lien très fort entre pointage vocal et pointage manuel qui se caractérise par une coordination geste / parole plus fine et plus systématique.

Bien que les travaux détaillés dans cet article concernent des études « contrôlées » menées dans des environnements non naturels et non de la parole spontanée enregistrée en milieu naturel, l'ensemble des résultats conforte la notion que le pointage serait de nature multimodale, non seulement dans son émergence chez les primates et dans son développement chez les bébés, mais aussi dans son expression dans la communication parlée adulte. 


\section{BIBLIOGRAPHIE}

Arbib M. A., 2003, The Evolving Mirror System: A Neural Basis for Language Readiness, in Christiansen M. \& Kirby S. (eds), Language Evolution: The States of the Art, Oxford University Press, Oxford, UK, p. 182-200.

Arbib M. A., 2005, From Monkey-Like Action Recognition to Human Language: An Evolutionary, Framework for Neurolinguistics, Behavioral and Brain Sciences 28(2), p. 105-124.

Arnold K. \& Zuberbühler K., 2006. Language evolution: semantic combinations in primate calls, Nature 441, 303 .

Astafiev S. V., Shulman G. L., Stanley C. M., Snyder A. Z., Van Essen D. C. \& Corbetta M., 2003, Functional Organization of Human Intraparietal and Frontal Cortex for Attending, Looking, and Pointing, Journal of Neuroscience 23, p. 4689-4699.

Astésano C., 2001, Rythme et accentuation en français: invariance et variabilité stylistique, Thèse de doctorat, L'Harmattan édition et diffusion.

Bates E., Benigni L., Bretherton I., Camaioni L. \& Volterra V., 1979, The emergence of symbols: cognition and communication in infancy, Academic Press, New York.

Butterworth G., 2003, Pointing is the Royal Road to Language for Babies, in S. Kita (ed), Pointing: Where Language, Culture, and Cognition Meet, Lawrence Erlbaum Associates : Hillsdale, NJ, p. 9-33.

Capirci O., Iverson J. M., Pizzuto E. \& Volterra V., 1996, Gestures and words during the transition to two-word speech, Journal of Child Language 23, p. $645-673$.

Caselli M. C., 1990, Communicative gestures and first words, in V. Volterra \& C. J. Erting (eds), From gesture to language in hearing and deaf children, Springer-Verlagn New York, p. 56-67.

Connolly J. D., Goodale M. A., Desouza J. F., Menon R. S. \& Vilis T., 2000, A comparison of frontoparietal fMRI activation during anti-saccades and antipointing, Journal of Neurophysiology 84, p. 1645-1655.

Corballis M. C., 2003. From Mouth to Hand: Gesture, Speech, and the Evolution of Right- Handedness. Behavioral and Brain Sciences, 26(2), p. 199-260.

Dahan D. \& Bernard J., 1996, Interspeaker Variability in Emphatic Accent Production in French, Language and Speech 39, p. 341-374

Delais-Roussarie E., Rialland A., Doetjes J., Marandin J. M., 2002, The Prosody of Post Focus Sequences in French, Proceedings of Speech Prosody 2002 p. 239-242.

Di Cristo A., 1998, Intonation in French, in D. Hirst \& A. Di Cristo (eds.), Intonation Systems: a Survey of Twenty Languages, Cambridge University Press, Cambridge, p. 195-218.

Di Cristo A., 2000, Vers une modélisation de l'accentuation du français (seconde partie), French Language Studies 10, p. 27-44.

Di Cristo A. \& Jankowski L., 1999, Prosodic organization and phrasing after focus in French, Proceedings of ICPhS 1999, p. 1565-1458.

Dohen M., 2005, Deixis prosodique multisensorielle: production et perception audiovisuelle de la focalisation contrastive en français, Thèse de doctorat en sciences cognitives, Grenoble-INP.

Dohen M. \& Lœvenbruck H., 2004, Pre-focal Rephrasing, Focal Enhancement and Post-focal Deaccentuation in French, Proceedings of the 8th 
International Conference on Spoken Language Processing (ICSLP 04), p. 785-788.

Dohen M. \& Lœvenbruck H., 2005, Audiovisual Production and Perception of Contrastive Focus in French: a multispeaker study, Proceedings of Interspeech 2005, p. 2413-2416.

Dohen M. \& Lœvenbruck H., 2009, Interaction of audition and vision for the perception of prosodic contrastive focus, Language and Speech 52(2-3), p. 177-206.

Dohen M., Lœvenbruck H. \& Hill H., 2006, Visual correlates of prosodic contrastive focus in French: description and inter-speaker variability, Proceedings of Speech Prosody 2006, p. 221-224.

Dohen M., Levenbruck H. \& Hill H., 2009, Recognizing Prosody from the Lips: Is It Possible to Extract Prosodic Focus from Lip Features, in A. Liew \& S. Wang (eds), Visual Speech Recognition: Lip Segmentation and Mapping, IGI Global, p. 416-438.

Feyereisen P., 1997, The Competition Between Gesture and Speech Production in Dual-Task Paradigms, Journal of Memory and Language 36(1), p. 13-33.

Flöel A., Ellger T., Breitenstein C. \& Knecht S., 2003, Language perception activates the hand motor cortex: implications for motor theories of speech perception, European Journal of Neuroscience 18, p. 704-708.

Fougeron C. \& Keating P. A., 1997, Articulatory strengthening at edges of prosodic domains, The Journal of the Acoustic Society of America 101, p. 3728-3740.

Goldin-Meadow S. \& Butcher C., 2003, Pointing Toward Two-Word Speech in Young Children, in S. Kita (ed.), Pointing: Where Language, Culture, and Cognition Meet, Lawrence Erlbaum Associates, Hillsdale, N.J., p. 85-107.

Gussenhoven C., 1983, Testing the Reality of Focus Domains, Language and Speech 26(1), p. 61-80.

Hagler D. J. Jr, Riecke L. \& Sereno M. I., 2007, Parietal and superior frontal visuospatial maps activated by pointing and saccades, Neurolmage 35 (4), p. $1562-1577$.

Halliday M. A. K., 1967, Intonation and grammar in British English, La Hague, Mouton.

Holden G., 2004, The Origin of Speech, Science 303, p. 1316-1319.

Hubbard A. L., Wilson S. M., Callan D. E. \& Dapretto M., 2009, Giving speech a hand: Gesture modulates activity in auditory cortex during speech perception, Human Brain Mapping 30, p. 1028-1037.

Iverson J. \& Thelen E., 2003, The Hand Leads the Mouth in Ontogenesis Too, Behavioral and Brain Sciences 26(2), p. 225-226.

Iverson J. \& Goldin-Meadow S., 1998, Why People Gesture as They Speak, Nature 396, 228.

Jun S.-A. \& Fougeron C., 2000, A phonological model of French intonation, in A. Botinis (ed), Intonation: Analysis, Modelling and Technology, Dordrecht,KAP, p. 209-242.

Kendon A., 2004, Gesture - Visible Action as Utterance, Cambridge, MA, Cambridge University Press.

Kertzman C., Schwarz U., Zeffiro T. A. \& Hallett M., 1997, The role of posterior parietal cortex in visually guided reaching movements in humans, Experimental Brain Research 114 (1), p. 170-183. 
Khaldoyanidi A. \& Morel M.-A., 2008, Prédication et focalisation à l'oral en russe et en français. Propriétés intonatives et mimico-gestuelles, Faits de langues 31-32, p. 227-242.

Lacquaniti F., Perani D., Guigon E., Bettinardi V., Carrozzo M., Grassi F., Rossetti Y. \& Fazio F., 1997, Visuomotor transformations for reaching to memorized targets: a PET study, Neuroimage 5 (2), p. 129-146.

Ladd R. D., 1996, Intonational Phonology, Cambridge, Cambridge University Press.

Lee K., Eskritt M., Symons L. A. \& Muir D., 1998, Children's Use of Triadic Eye Gaze Information for "Mind Reading", Developmental Psychology 34(3), p. 525-539.

Lemasson A. \& Barbu S. (présent volume), L'origine phylogénétique du langage: apports des travaux récents sur la communication vocale des cercopithèques, Faits de langues 37.

Levelt W. J. M., Richardson G. \& Heij W. L., 1985, Pointing and Voicing in Deictic Expressions, Journal of Memory and Language 24, p. 133-164.

Lœvenbruck H., 1999, An investigation of articulatory correlates of the Accentual Phrase in French, Proceedings of the XIVth International Congress of Phonetic Sciences, p. 667-670.

Lœvenbruck H., Baciu M., Segebarth C. \& Abry C., 2005, The left inferior frontal gyrus under focus: an fMRI study of the production of deixis via syntactic extraction and prosodic focus, Journal of Neurolinguistics 18, p. $237-258$.

McNeill D., 1992, Hand and Mind, Chicago, University of Chicago Press.

McNeill D., 2000, Language and gesture, Cambridge, Cambridge University Press.

MacNeilage P. F., 1998, The Frame/Content Theory of Evolution of Speech Production, Behavioral and Brain Sciences 21(4), p. 499-511.

MacNeilage P. F. \& Davis B., 2000, On the Origin of Internal Structure and Word Forms, Science 288, p. 527-531.

Morel M.-A. \& Danon-Boileau L. (dir.), 1992, La deixis, Colloque en Sorbonne, Paris, PUF.

Morford M. \& Goldin-Meadow S., 1992, Comprehension and production of gesture in combination with speech in one-word speakers, Journal of Child Language 19 (3), p. 559-580.

Nølke H., 1994, Linguistique modulaire : de la forme au sens, Louvain, Peeters.

Özyürek A., Willems R. M., Kita S. \& Hagoort P., 2007, On-line integration of semantic information from speech and gesture: Insights from event-related brain potentials, Journal of Cognitive Neuroscience 19(4), p. 605-616.

Pizzuto E., Capobianco M. \& Devescovi A., 2005, Gestural-Vocal Deixis and Representational Skills in Early Language Development, Interaction Studies 6(2), p. 223-252.

Rochet-Capellan A., 2007, De la substance à la forme: rôle des contraintes motrices orofaciales et brachiomanuelles de la parole dans l'émergence du langage, Thèse de doctorat de sciences cognitives, Grenoble-INP.

Rochet-Capellan A., Schwartz J.-L., Laboissière R. \& Galván A., 2008, The Speech Focus Position Effect on Jaw-Finger Coordination in a Pointing Task, Journal of Speech, Language and Hearing Research 51(6), p. 1507-1521.

Rossi M., 1999, La focalisation, in L'intonation, le système du français : description et modélisation (Chapitre II-6, p. 116-128), Paris, Ophrys. 
Simon O., Mangin J. F., Cohen L., Le Bihan D. \& Dehaene S., 2002, Topographical layout of hand, eye, calculation, and language-related areas in the human parietal lobe, Neuron 33, p. 475-487.

Slocombe K. E., \& Zuberbühler K., 2006, Food-associated calls in chimpanzees: Responses to food types or food preferences?, Animal Behaviour 72(5), p. 989-999.

Tabain M., 2003, Effects of prosodic boundary on /aC/ sequences: Articulatory results, The Journal of the Acoustical Society of America 113 (5), p. 28342849.

Touati P., 1987, Structures prosodiques du suédois et du français, Working Paper 21. Lund University Press.

Vallar G., Guariglia C., Nico D. \& Pizzamiglio L., 1997, Motor deficits and optokinetic stimulation in patients with left hemineglect, Neurology 49 (5), p. 1364-1370.

Vauclair J., (présent volume), Du geste à la parole. De la communication des primates au langage humain, Faits de langues 37.

Volterra V., Caselli M. C., Capirci O. \& Pizzuto E., 2005, Gesture and the Emergence and Development of Language, in M. Tomasello \& D. Slobin (eds), Elizabeth Bates: A Festschrift, Mahwah, NJ., Lawrence Erlbaum Associates, p. 3-40

Willems R. M., Özyürek A. \& Hagoort P., 2007, When language meets action: The neural integration of gesture and speech, Cerebral Cortex 17, p. 23222333.

\section{ANNEXE}

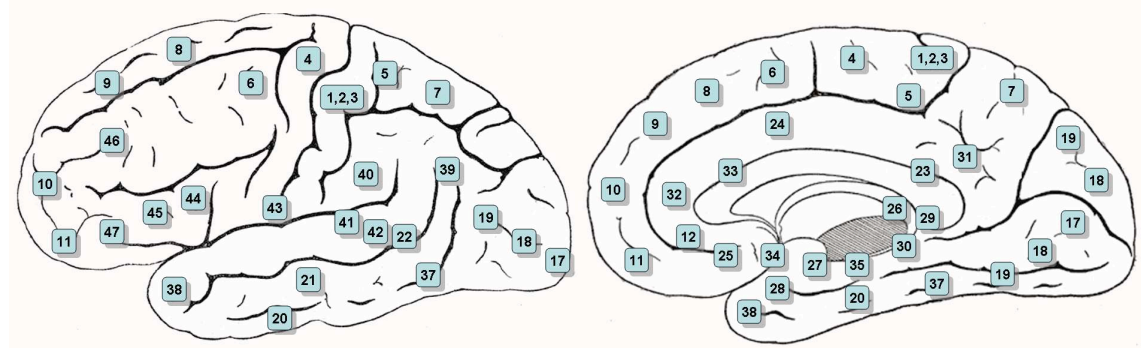

Cartographie des aires de Brodmann : surfaces latérale (à gauche) et médiane (à droite) de l'hémisphère gauche.

[source : http://fr.wikipedia.org/wiki/Aires_de_Brodmann] 